angles. This approximation is then made closer by using the values of $f$ at points where $A B$ cuts the curve $f(x, y)=$ const. If the second approximation is not close enough, the process is repeated.

23. Herr Wagenmann correlates successive steps in the theory of evolution with series $-\infty, \cdots-2,-1,0,1,2, \cdots, \infty$ along three coördinate axes developing successively the ideas of motion, mass, the nebular hypothesis and evolution of living organisms and of civilization. He finds that his method leads to a monistic philosophy - in fact to a pan-monism.

\title{
Göttingan,
}
A. B. Frizeil.

November, 1906.

\section{A NEW APPROXIMATE CONSTRUCTION FOR $\pi$.}

BY MR. GEORGE PEIRCE.

Given a circle with radius $r$ and center at $O$; to find an approximate construction for $\pi r$.

Draw the diameter $A O B$ and the tangent $B C$ at right angles to it. Describe the arc $O D C$ with radius $r$ and center at $B$.

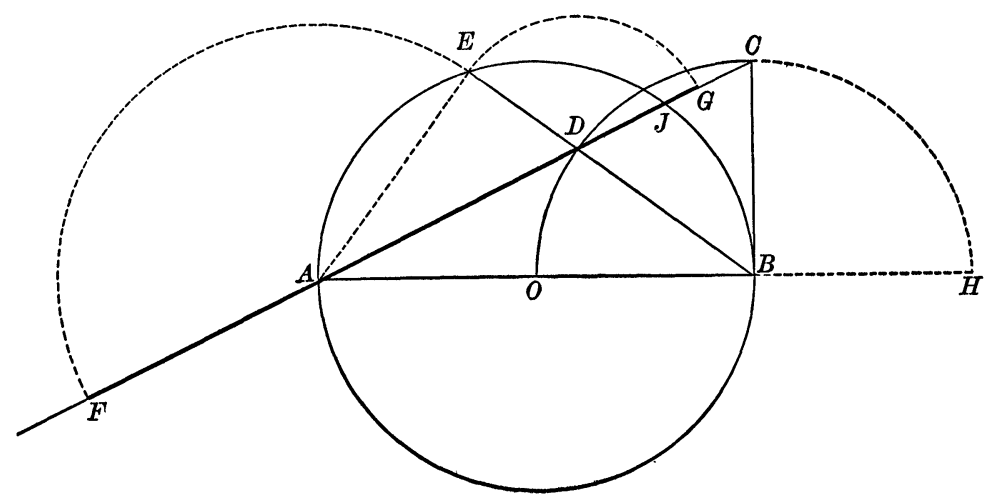

Draw the line $A C$ cutting the arcs $O D C$ and $A B$ at $D$ and $J$; also draw the line $B D E$ through $B$ and $D$ cutting the given circle at $E$. Then $A D+3 D E=\pi r$ approximately. 
Proof :

$$
\begin{gathered}
A C=\sqrt{\left(\overline{A B^{2}+B C^{2}}\right)=r \sqrt{5}} \\
A D=\frac{A O \cdot A H}{A C}=\frac{r \cdot 3 r}{r \sqrt{5}}=\frac{3}{5} \sqrt{5} r \\
J C=\frac{B C^{2}}{A C}=\frac{r^{2}}{r \sqrt{5}}=\frac{1}{5} \sqrt{5} r \\
\left.D J=A C-A D-J C=\frac{1}{5}\right\urcorner \overline{5} r \\
D E=\frac{A D \cdot D J}{B D}=\frac{\frac{3}{5} \sqrt{5} r \cdot \frac{1}{5} \sqrt{5} r}{r}=\frac{3}{5} r \\
A D+3 D E=\frac{3}{5} \sqrt{5} r+3\left(\frac{3}{5} r\right)=3.141641 r
\end{gathered}
$$

By making use of the fact that in the triangle $A B E$

$$
A E=\sqrt{\left(A B^{2}-B E^{2}\right)}=\sqrt{(2 r)^{2}-\left(\frac{8}{6} r\right)^{2}}=\frac{6}{b} r=2 D E,
$$

we can obtain a single line of the same length as $A D+3 D E$. We can therefore draw the arc $E G$ with radius $D E$ and center at $D$ and the arc $E F$ with radius $A E$ and center at $A$. Then $A D+3 D E=A D+A E+D E=A D+F A+D J=F G$.

There are many other approximate constructions for $\pi r$. A summary of those that have been worked out according to the method of geometrography is given below. $A, B, C$ and $D$ are to be found in the Bulletin for January, 1902, page 137 ; $E$ is in Cantor's Geschichte der Mathematik, volume 3, page 23; $F$ is the construction given above.

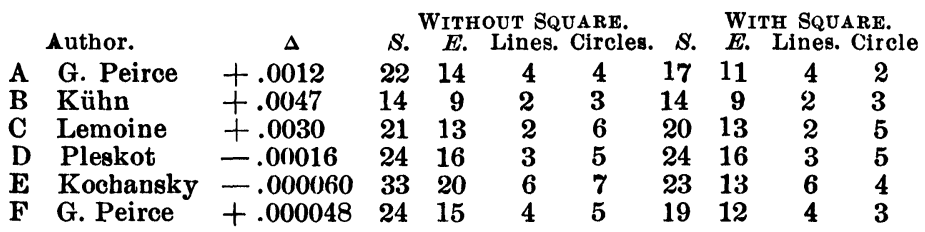

$\Delta$ is the difference between the mechanically exact construction and $\pi$. $S$ stands for simplicity and $E$ for exactitude. For the technical meanings of these two words see the article in the Bulletis for January, 1902. The lower these numbers are, the better the construction. 